\title{
Risk factors for adverse events induced by immune checkpoint inhibitors in patients with non-small-cell lung cancer: a systematic review and meta-analysis
}

\author{
E. Suazo-Zepeda ${ }^{1} \cdot$ M. Bokern ${ }^{1} \cdot$ P. C. Vinke ${ }^{1} \cdot$ T. J. N. Hiltermann ${ }^{2} \cdot$ G. H. de Bock ${ }^{1} \cdot$ G. Sidorenkov $^{1}$ (D)
}

Received: 14 February 2021 / Accepted: 19 June 2021 / Published online: 30 June 2021

(c) The Author(s) 2021

\begin{abstract}
Background Immune checkpoint inhibitors (ICIs) can cause serious immune-related adverse events (irAEs). This study aimed to identify risk factors for all types of irAEs induced by ICIs in patients with non-small-cell lung cancer (NSCLC), by systematic review and meta-analyses.

Methods A systematic search was performed in Pubmed, Embase and Web of Science by two independent reviewers. Studies were selected that included patients with NSCLC and evaluated characteristics of patients with and without irAEs induced by ICIs. Quality and risk of bias of the selected studies were assessed. Random effects meta-analyses were conducted to estimate pooled odds ratios (ORs) for risk factors of developing all type of irAEs, and separately for pneumonitis, interstitial lung disease and severe irAEs. With the objective of exploring sources of heterogeneity, stratified analyses were performed by quality and region.

Results 25 studies met the inclusion criteria. In total, the data of 6696 patients were pooled. 33 different risk factors for irAEs were reported. irAEs of interest were reported for 1653 (25\%) of the patients. Risk factors related to the development of irAEs were: C-reactive protein, neutrophil lymphocyte ratio (NLR), use of PD-1 inhibitor, high PD-L1 expression, an active or former smoking status, ground glass attenuation, and a better treatment response.

Conclusion The identified risk factors for the development of these irAEs are mostly related to the alteration of the immune system, proinflammatory states and loss of immunological self-tolerance. Patients identified as having a higher risk for irAEs should be monitored more closely.
\end{abstract}

Keywords Lung cancer $\cdot$ Immunotherapy $\cdot$ Adverse events $\cdot$ Immune checkpoint inhibitors

\section{Introduction}

Lung cancer is one of the most commonly diagnosed cancers in the world, as well as the leading cause of cancer death in men and women [1]. It ranks as one of the cancers with the lowest survival, being about $50 \%$ within 1 year after diagnosis, and $20 \%$ within 5 years [2]. Of all lung cancer cases, more than $85 \%$ percent are classified as non-small-cell lung

G. Sidorenkov

g.sidorenkov@umcg.nl

1 Department of Epidemiology, Graduate School of Medical Sciences, University of Groningen, Zusterhuis, Hanzeplein 1, Groningen, The Netherlands

2 Department of Pulmonology, University of Groningen, University Medical Center Groningen, Groningen, Netherlands cancer (NSCLC), and treatment efforts have mainly focused on this histological type [3].

In recent years, new treatments have emerged as an effective option for patients diagnosed with advanced NSCLC. Patients with advanced NSCLC especially benefit from the introduction of immunotherapy with immune checkpoint inhibitors (ICIs), which have shown better efficacy and safety compared to older treatments like chemotherapy and radiotherapy [4]. At present, the ICIs that target programmed cell death 1 (PD-1)/programmed cell death-ligand 1 (PD-L1) and the cytotoxic T-lymphocyte-associated protein 4 (CTLA 4), allowing the activation of the T lymphocytes antineoplastic activity, are approved by the Food and Drug Administration (FDA) for the treatment of advanced NSCLC [5, 6]. The ICIs can cause immune-related adverse events (irAEs), the incidence of which, depending on their degree, varies between 24 and 38\% [7-12]. These irAEs are 
due to the alteration of immunological self-tolerance caused by the blockade of the immune checkpoint receptors PD-1/ PD-L1 and CTLA-4, and are different from adverse events (AEs) after chemotherapy and radiotherapy [13]. The skin, liver, gastrointestinal, pulmonary and endocrine organs are most commonly affected $[14,15]$.

The occurrence of severe irAEs requiring immunosuppression and complete cessation of ICI treatment is estimated between 9 and 33\% [7-11]. The high cost of these therapies and the severity of the irAEs create a need to identify risk factors for AEs of immunotherapy that can be taken into account when choosing a therapy for patients with NSCLC [16]. Previous studies described AEs induced by ICIs [17, 18], and other studies that evaluated characteristics that predispose patients treated with ICIs to irAEs as well [19-21]. Although previous systematic reviews have evaluated risk factors for adverse events, they either focus on a limited number of risk factors (like tumor or ICI class) [20], or on selected adverse events, such as fatal toxicities [21]. Therefore, we aimed to review and meta-analyze the results of published studies to identify risk factors for irAEs, not limited to specific risk factor domains or types of irAEs. Since irAE patterns do differ per tumor type [20], we restricted this review to studies reporting data on patients with NSCLC.

\section{Methods}

\section{Review registration}

This systematic review was registered in the PROSPERO International prospective register of systematic reviews with the registration number CRD42020194101. The research protocol was prepared following the Guidance notes for registering a systematic review protocol with PROSPERO [22].

\section{Data sources and researches}

Three databases were searched, Pubmed, Embase and Web of Science. Studies published in English between January 1st, 2000 and November 12th, 2020 were reviewed. Key search terms in developing the search strategy were the following: lung cancer, lung neoplasms, immunomodulators, immune checkpoint inhibitors, and specific drug names of ICIs (nivolumab, pembrolizumab, atezolizumab, durvalumab, cemiplimab, avelumab). The search strategy is presented in Supplementary material 1.

\section{Study selection}

Studies were included if the following inclusion criteria were met: (1) study was designed as a randomized clinical trial, cohort or case-control study, (2) patients were diagnosed with NSCLC, and (3) treated with ICIs (4) the study evaluated characteristics of patients presenting adverse events induced by ICIs, specifically PD-1/PDL-1 and CTLA-4 protein blockers. Studies were excluded, (1) if these only evaluated the safety and effectiveness of ICIs and did not evaluate the relationship between risk factors and irAEs; or (2) included less than 10 participants. Three reviewers (ES-Z, MB, GS) performed an initial review and selection of the publications independently according to their title and abstract. Subsequently, two reviewers (ES-Z, MB) read the full text of these publications for their final inclusion. Disagreements in study selection were mediated by a third reviewer (GS).

\section{Data extraction}

The main outcome was the occurrence of any type of immunotherapy-induced adverse events (irAEs), defined as all disorders with a potential immunological background that resulted from the use of ICIs. Included in this definition were a broad variety of chemically induced adverse conditions caused by toxicity, drug interactions, and metabolic events of ICIs.

The Cochrane data collection form [23] (Supplementary material 2) adapted to our study and research question was used independently by the reviewers (MB and ES-Z) to collect data from the selected publications, which was always checked by a third reviewer (PCV). The following information was extracted: first author, year of publication, region, publication type, study funding source, study design, inclusion and exclusion criteria, total number of patients, outcomes (type of irAEs, e.g., ILD or pneumonitis), secondary outcomes (common irAEs, e.g., skin reactions or mucositis), risk factors, methods of recruitment of participants, follow-up time, irAEs reporting rate, and the frequency of each specific grade AE. The odds ratio (OR) for each risk factor was retrieved from the studies. When the OR adjusted for confounders was not available, the crude OR was used or calculated based on the frequency tables (if available). If indicated, ORs and confidence intervals were reversed rising the value to the -1 power $\left(\mathrm{OR}^{-1}\right.$ [Lower $\mathrm{CI}^{-1}$; Upper $\left.\mathrm{CI}^{-1}\right]$ ) to harmonize the reference categories within each risk factor.

\section{Quality assessment}

Two reviewers (MB and ES-Z) assessed the quality and risk of bias of the selected papers using the Cochrane Collaboration's tool for assessing risk of bias in the case of Randomized clinical trials, or the Quality in Prognostic Studies (QUIPS) [24] in the case of non-randomized studies (case-control studies and cohort studies). The "Scottish 
Intercollegiate Guidelines Network: rating a quality of Cohort Studies" was used to classify papers into high quality, acceptable quality and low quality. Disagreements in the study selection, data collection, and quality assessment steps were mediated by a third reviewer (GS) and discussed until consensus was reached.

\section{Data synthesis and analysis}

Study characteristics as well as study outcomes were described. When at least two studies reported data on the same risk factor, random effects meta-analysis models were performed including both crude and adjusted ORs to provide pooled ORs and the related $95 \%$ confidence intervals $(95 \% \mathrm{CI})$, where the outcome was the occurrence of any irAEs. A stratified analysis was performed for interstitial lung disease (ILD), pneumonitis, severe irAEs. The included studies classified as severe those irAEs grade 3 or higher according to the Common Terminology Criteria for Adverse Events (CTCAE). This pooling was done under the assumption of homogeneity. To evaluate the justification of this assumption, the $\chi^{2}$ test statistic $I^{2}$ was calculated. A value of $I^{2}>50 \%$ was considered as heterogeneous. To explore sources of heterogeneity, stratified analyses were performed by quality/risk of bias (high, moderate and low risk of bias), and study region (Asia vs USA/Europe). Sensitivity analyses were performed by excluding the crude ORs to assess the validity of the meta-analysis. Analyses were performed using Stata software, version 14.0 (StataCorp).

\section{Results}

From the three databases, after deleting duplicates, 221 potentially eligible articles were obtained for title and abstract screening after deleting duplicates (Supplementary Fig. 1). After the initial screening, 45 articles were selected for full text reading, of which 25 met the inclusion and exclusion criteria. The reasons for exclusion were: 11 studies did not stratify by type of cancer (NSCLC/ other cancers), one study included less than 10 patients, six studies did not assess risk factors for adverse events, one study did not stratify by type of treatment (chemotherapy/ immunotherapy) and one study included the same group of patients and risk factors twice.

A total of 6696 unique patients were included in all studies. The number of patients included per study ranged from 42 to 1548 (Table 1). 14 studies took place in Japan (1859 patients), seven in the United States of America (USA) (3179 patients), two in China (366 patients) and two in Europe (1292 patients). irAEs of interest were reported for 1653 (25\%) of the patients. 33 different risk factors for irAEs were reported by the studies included in the meta-analyses.
The most frequently reported risk factors were sex (19 studies), ECOG performance status (16 studies), histology (16 studies), smoking status (13 studies) and age (12 studies) (Supplementary able 1). The included studies focused on the following irAEs: all types of irAEs combined (8 studies), ILD (6 studies), pneumonitis (5 studies), severe irAEs (3 studies), acute exacerbation of ILD (1 study), infectious diseases ( 1 study), skin reactions ( 1 study), immune-related thyroid dysfunction (1 study), ICI-related cardiotoxicity (1 study) (see Table 1).

Six studies were graded as having high quality/low risk of bias, eight as having moderate quality/risk of bias and eleven as having low quality/high risk of bias (Supplementary Fig. 2). Low quality papers had important problems in statistical analysis and reporting problems (9 studies) or did not provide a clear definition of confounders or adjustment (8 studies).

In the meta-analyses combining the crude and adjusted ORs, the following risk factors were significantly associated with the development of irAEs: C-reactive protein (OR 1.08; CI 95\% 1.007-1.158); neutrophil lymphocyte ratio (NLR) (OR 1.046; CI 95\% 1.006-1.088), the presence of ground glass attenuation in CT imaging (OR 77.1; CI 95\% 7.82-760.3), use of PD-1 inhibitor agent (OR 2.39, CI 95\% 1.073-5.326), higher PD-L1 expression (OR 2.009, CI 95\% 1.03-3.921), an active or former smoking status (OR 1.249; CI 95\% 1.021-1.528), and a better treatment response (OR 3.548; CI 95\% 2.49-5.055) (Table 2; Fig. 1).

In the sensitivity analyses excluding the crude ORs, 10 studies were included [25-34]. Risk factors that could be pooled and were significantly related with the development of irAEs were squamous histology (OR 1.847; CI 95\% 1.048-3.256), a higher number of pack-years of smoked tobacco (OR 2.662; CI 95\% 1.014-6.989) and the presence of ground glass attenuation in CT imaging (OR 77.1; CI 95\% 7.82-760.3) (Supplementary Table 2).

As part of the stratified meta-analyses by type of irAE (Supplementary Table 3), it was observed that men compared to women are at higher risk of developing ILD (OR 1.79; CI 95\% 1.054-3.041). In the case of pneumonitis, no significant associations were found with the tested risk factors (Supplementary Table 3). For irAEs classified as severe, patients with a worse ECOG performance status are at higher risk (OR 4.274; CI 95\% 1.215-14.925; Supplementary Table 3 ).

For risk factors with significant heterogeneity $\left(I^{2}>50 \%\right)$, additional analyses stratified by quality/risk of bias and region were performed to explore sources of heterogeneity. For stage and combination treatment (ICI + chemotherapy vs. monotherapy), quality/risk of bias of included studies was an explanatory factor for the observed heterogeneity in the pooled analyses. For these risk factors, there was no longer heterogeneity when only moderate or high quality 
Table 1 Study characteristics

\begin{tabular}{|c|c|c|c|c|}
\hline Author & Year & Location & Participants & Outcome \\
\hline Ahmed T [45] & 2020 & USA & 285 & All type of irAEs \\
\hline Aso M [46] & 2020 & Japan & 155 & Skin reactions \\
\hline Chu X [27] & 2020 & China & 300 & Pneumonitis \\
\hline Cortellini A [47] & 2020 & Italy & 1010 & All type of irAEs \\
\hline Duma N [34] & 2019 & USA & 231 & All type of irAEs, severe irAEs \\
\hline Fujita K [26] & 2019 & Japan & 167 & Infectious diseases \\
\hline
\end{tabular}

Fukihara J [48] $\quad 2019$ Japan $\quad 170 \quad$ Pneumonitis

$1548 \quad$ All type of irAEs

Type of risk factor

Performance status, age

Histology, PD-L1 expression, performance status, preexisting anti-nuclear antibodies, anti-thyroid antibodies, rheumatoid factor, sex, smoking status

Age, sex, smoking status, performance status, treatment line, treatment combination, preexisting lung diseases, use of steroids, eosinophil count

Sex, age, performance status, histology, number of organs affected

Sex

Age, ALK gene, asthma, bronchiectasis, COPD, corticosteroids before nivolumab, corticosteroids during/after nivolumab, diabetes EGFR mutation, histology, history of thoracic surgery, hypothyroidism, ILD, initial stage no. cycles, performance status, radiotherapy, rheumatoid arthritis, sex, smoking status

Abnormal CT findings, age, albumin, ALT, AST, creatinine CRP, emphysema, histology, ILD, NLR, past thoracic RT, pembrolizumab treatment, performance status, sex, smoking pack-years, treatment line, white blood cell count

Sex, ethnicity, performance status, histology, treatment line, smoking status, PD-L1 positive vs negative, $\mathrm{LDH}$, lung immune prognostic index

Koyama J [25] 2019 Japan

132

Metro G [50]

Moey M [51]

2020 Greece, Italy, Spain, Switzerland

2020 USA
282

196
Inhibitor-related cardiotoxicity
Immune-related thyroid dysfunction

All type of irAEs

Age, EGFR/ALK positive, histology PD-L1 expression, performance status, sex, smoking status, thyroid dysfunction, treatment line, TTF1 expression

Brain metastasis

Ethnicity, concomitant irAEs, COPD, diabetes, hyperlipidemia, hypertension, PD-(L)1 agent, previous atrial fibrillation/flutter, previous cardiovascular disease, previous cerebrovascular disease, previous chronic kidney disease, radiotherapy, sex, stage, type of lung tumor (NSCLC), use of alkylating agents, use of anti-topoisomerase drugs, use of anti-VEGF drugs, use of antimetabolites, use of betablocker, use of calcium channel blocker, use of loop diuretics, use of renin-angiotensinaldosterone system inhibitors, use of statins, use of steroids, use of taxanes, use of thiazide diuretic

CRP, histology, IAOT, LDH, performance status, pleural effusion, previous pneumonitis pulmonary metastasis, sex, smoking status 
Table 1 (continued)

\begin{tabular}{|c|c|c|c|c|c|}
\hline Author & Year & Location & Participants & Outcome & Type of risk factor \\
\hline Nakanishi Y [29] & 2019 & Japan & 83 & ILD & $\begin{array}{l}\text { Abnormal CT findings, age CRP, driver muta- } \\
\text { tion, emphysema, ground glass attenuation, } \\
\text { histology history of radiation pneumonitis, } \\
\text { honeycombing, KL-6 in serum, LDH, lym- } \\
\text { phocyte count, neutrophil count, NLR, PD-L1 } \\
\text { expression, performance status, preexisting } \\
\text { ILD, prior thoracic RT, reticular shadow, sex, } \\
\text { smoking status, white blood cell count }\end{array}$ \\
\hline Nishiyama N [28] & 2019 & Japan & 48 & Acute exacerbation of ILD & $\begin{array}{l}\text { Age ground glass attenuation, performance sta- } \\
\text { tus, sex, smoking pack-years, UIP radiological }\end{array}$ \\
\hline Okada N [32] & 2020 & Japan & 102 & ILD & $\begin{array}{l}\text { COPD, histology, ILD, PD-(L)1 agent, PD-L1 } \\
\text { expression } \geq 50 \% \text { vs }<50 \% \text {, performance } \\
\text { status, radiotherapy, sex, smoking pack-years, } \\
\text { stage, treatment line, use of EGFR-TKI }\end{array}$ \\
\hline Owen DH [52] & 2018 & USA & 91 & Pneumonitis & $\begin{array}{l}\text { ALI, heavy smoking history, histology, KRAS } \\
\text { mutation, NLR non-CNS radiation after ICI, } \\
\text { PLR, RT after ICI, sex, smoking status, tho- } \\
\text { racic/chest wall RT }\end{array}$ \\
\hline Sakata Y [53] & 2019 & Japan & 42 & Severe irAEs & $\begin{array}{l}\text { Age, histology, initial stage PD-L1 expression, } \\
\text { pembrolizumab treatment, performance status, } \\
\text { sex, smoking status, treatment line, tumor } \\
\text { burden }\end{array}$ \\
\hline Shankar B [54] & 2020 & USA & 623 & All type of irAEs & $\begin{array}{l}\text { Histology, non-white ethnicity, PD-(L)1 agent, } \\
\text { performance status, sex, smoking status, stage, } \\
\text { treatment combination, treatment response }\end{array}$ \\
\hline Sugano T [31] & 2020 & Japan & 130 & ILD & $\begin{array}{l}\text { Age, histology, performance status, previous } \\
\text { interstitial pneumonia, radiotherapy, sex, } \\
\text { smoking status }\end{array}$ \\
\hline Suresh K [55] & 2018 & USA & 205 & Pneumonitis & $\begin{array}{l}\text { Age, ethnicity, chemotherapy, combination ICI } \\
\text { treatment, histology, initial stage, other treat- } \\
\text { ments, pembrolizumab treatment, ethnicity, } \\
\text { sex, smoking status, surgery }\end{array}$ \\
\hline Suzuki Y [56] & 2020 & Japan & 138 & ILD & $\begin{array}{l}\text { Asthma, best supportive care after ICIs, COPD, } \\
\text { diabetes, digestive ulcer, hepatic disease, his- } \\
\text { tology, hypertension, PD-L1 expression } \geq 50 \% \\
\text { vs }<50 \% \text {, performance status, previous car- } \\
\text { diovascular disease, previous cerebrovascular } \\
\text { disease, sex, smoking status, stage, treatment } \\
\text { line, treatment response }\end{array}$ \\
\hline Toi Y [57] & 2019 & Japan & 137 & All type of irAEs & $\begin{array}{l}\text { Histology, performance status, preexisting anti- } \\
\text { nuclear, antibodies, anti-thyroid antibodies, } \\
\text { rheumatoid factor, sex, smoking status }\end{array}$ \\
\hline Watanabe S [58] & 2020 & Japan & 231 & ILD & $\begin{array}{l}\text { Cryptogenic organizing pneumonia-like, } \\
\text { histology, PD-L1 expression } \geq 50 \% \text { vs }<50 \% \text {, } \\
\text { pembrolizumab treatment, performance status, } \\
\text { sex, smoking status, stage, treatment line }\end{array}$ \\
\hline Yamaguchi T [33] & 2018 & Japan & 123 & Pneumonitis & $\begin{array}{l}\text { Age, CRP, emphysema score, fibrosis, histology, } \\
\text { LDH performance status, sex, smoking status, } \\
\text { treatment line }\end{array}$ \\
\hline Zeng X [59] & 2020 & China & 66 & All type of irAEs & Comorbidity index \\
\hline
\end{tabular}

$P D-L 1$ programmed death-ligand 1; $A L K$ anaplastic lymphoma kinase; $C O P D$ chronic obstructive pulmonary disease; EGFR epidermal growth factor receptor; NSCLC non-small-cell lung carcinoma; irAEs immune-related adverse events; ILD interstitial lung disease; $C R P C$-reactive protein; $C T$ computed tomography; $A L T$ alanine transaminase; $A S T$ aspartate transaminase; $N L R$ neutrophil to lymphocyte ratio; $R T$ radiotherapy; $T T F 1$ transcription termination factor 1; $L D H$ lactate dehydrogenase; ICI immune checkpoint inhibitors; UIP usual interstitial pneumonia; CNS central nervous system; PLR platelet lymphocyte ratio; IAOT imaging findings of airway obstruction adjacent to lung tumors; VEGF vascular endothelial growth factor 
Table 2 Random effects meta-analysis. Pooled crude and adjusted odds ratios reflecting the association between risk factors and irAEs in patients treated with ICIs

\begin{tabular}{|c|c|c|c|c|c|c|c|c|c|}
\hline Risk factor & Pooled OR & LCI & UCI & Chi-2 & Chi-2 $p$ value & $\mathrm{I} 2$ & $P$ value & No. of studies & $\begin{array}{l}\text { No. of } \\
\text { partic- } \\
\text { ipants }\end{array}$ \\
\hline $\begin{array}{l}\text { Abnormal CT findings } \\
\text { (abnormal CT vs normal } \\
\text { CT) }\end{array}$ & 1.193 & 0.607 & 2.343 & 1.04 & 0.307 & $4.20 \%$ & 0.609 & $2[29][48]$ & 253 \\
\hline Age $(\geq 65$ vs $<65)$ & 1.124 & 0.904 & 1.396 & 11.43 & 0.178 & $30 \%$ & 0.292 & $\begin{array}{c}10[25][26][27][28][31] \\
{[33][45][47][53][55]}\end{array}$ & 2442 \\
\hline Age (years) & 0.988 & 0.954 & 1.023 & 0.39 & 0.533 & $0.00 \%$ & 0.49 & $2[29][48]$ & 253 \\
\hline $\begin{array}{l}\text { Asthma (history of asthma vs } \\
\text { no history of asthma) }\end{array}$ & 1.729 & 0.226 & 13.209 & 0.87 & 0.35 & $0.00 \%$ & 0.598 & $2[26][56]$ & 305 \\
\hline $\begin{array}{l}\text { Black ethnicity (black vs } \\
\text { white ethnicity) }\end{array}$ & 0.709 & 0.346 & 1.456 & 1.42 & 0.233 & $29.50 \%$ & 0.349 & $2[51][55]$ & 401 \\
\hline $\begin{array}{l}\text { COPD (history of COPD vs } \\
\text { no history of COPD) }\end{array}$ & 1.439 & 0.983 & 2.108 & 5.21 & 0.391 & $3.90 \%$ & 0.061 & $6[26][29][32][48][51][56]$ & 853 \\
\hline $\mathrm{CRP}(\mathrm{mg} / \mathrm{dL})$ & 1.08 & 1.007 & 1.158 & 0.08 & 0.779 & $0.00 \%$ & 0.03 & $2[29][48]$ & 253 \\
\hline $\begin{array}{l}\text { Diabetes (history of diabetes } \\
\text { vs no history of diabetes) }\end{array}$ & 1.672 & 0.86 & 3.248 & 2.8 & 0.247 & $28.50 \%$ & 0.129 & $3[26][51][56]$ & 501 \\
\hline $\begin{array}{l}\text { Ground glass attenuation } \\
\text { (ground glass attenuation vs } \\
\text { normal CT) }\end{array}$ & 77.098 & 7.818 & 760.304 & 0.88 & 0.349 & $0.00 \%$ & 0.0001 & 2 [28] [29] & 131 \\
\hline $\begin{array}{l}\text { Histology (squamous vs non- } \\
\text { squamous) }\end{array}$ & 0.934 & 0.806 & 1.082 & 23.33 & 0.077 & $35.70 \%$ & 0.363 & $\begin{array}{l}16[25-27,29-33,46-49,53 \\
\quad 54,56,58]\end{array}$ & 5155 \\
\hline $\begin{array}{l}\text { Hypertension (history of } \\
\text { hypertension vs no history } \\
\text { of hypertension) }\end{array}$ & 0.508 & 0.247 & 1.048 & 0.52 & 0.471 & $0.00 \%$ & 0.067 & $2[51,56]$ & 334 \\
\hline $\begin{array}{l}\text { ILD (previous history of ILD } \\
\text { vs no history of ILD) }\end{array}$ & 1.174 & 0.479 & 2.876 & 1.41 & 0.493 & $0.00 \%$ & 0.726 & $3[26,32,48]$ & 439 \\
\hline $\mathrm{LDH}(\geq 240$ vs $<240 \mathrm{U} / \mathrm{L})$ & 1 & 0.999 & 1.001 & 7.03 & 0.071 & $57.30 \%$ & 0.749 & $4[29,30,33,49]$ & 1955 \\
\hline $\operatorname{NLR}(\geq 5$ vs $<5)$ & 1.046 & 1.006 & 1.088 & 0.26 & 0.879 & $0.00 \%$ & 0.026 & 3 [29] [48] [52] & 344 \\
\hline $\begin{array}{l}\text { PD-(L)1 agent (PD-1 vs } \\
\text { PD-L1 inhibitor) }\end{array}$ & 2.39 & 1.073 & 5.326 & 0.56 & 0.755 & $0.00 \%$ & 0.033 & $3[32][51][54]$ & 921 \\
\hline $\begin{array}{l}\text { PD-L1 expression }(\geq 50 \% \\
\text { vs }<50 \%)\end{array}$ & 2.009 & 1.03 & 3.921 & 0.33 & 0.954 & $0.00 \%$ & 0.041 & $4[32][53][56][58]$ & 513 \\
\hline $\begin{array}{l}\text { PD-L1 expression (positive vs } \\
\text { negative) }\end{array}$ & 0.996 & 0.791 & 1.254 & 0 & 0.991 & $0.00 \%$ & 0.973 & 2 [25] [49] & 1680 \\
\hline $\begin{array}{l}\text { Pembrolizumab treatment } \\
\text { (pembrolizumab treatment } \\
\text { vs other ICIs) }\end{array}$ & 1.586 & 0.934 & 2.693 & 8.45 & 0.037 & $64.50 \%$ & 0.088 & $4[48][53][55][58]$ & 648 \\
\hline $\begin{array}{l}\text { Performance status ( } \geq 2 \text { vs } \\
0-1 \text { ) }\end{array}$ & 0.801 & 0.655 & 0.979 & 25.3 & 0.088 & $32.80 \%$ & 0.055 & $\begin{array}{l}18[25-33,45-49,53,54, \\
56,58]\end{array}$ & 5488 \\
\hline $\begin{array}{l}\text { Preexisting anti-nuclear } \\
\text { antibodies (preexisting } \\
\text { anti-nuclear antibodies vs } \\
\text { no preexisting anti-nuclear } \\
\text { antibodies) }\end{array}$ & 1.524 & 0.857 & 2.709 & 1.54 & 0.215 & $35.00 \%$ & 0.151 & $2[27][46]$ & 455 \\
\hline $\begin{array}{l}\text { Previous cardiovascular dis- } \\
\text { ease (history of cardiovas- } \\
\text { cular disease vs no history } \\
\text { of cardiovascular disease) }\end{array}$ & 2.295 & 0.93 & 5.663 & 2.26 & 0.133 & $55.70 \%$ & 0.072 & $2[51][56]$ & 334 \\
\hline $\begin{array}{l}\text { Previous cerebrovascular dis- } \\
\text { ease (history of cerebrovas- } \\
\text { cular disease vs no history } \\
\text { of cerebrovascular disease) }\end{array}$ & 0.905 & 0.277 & 2.959 & 0.76 & 0.383 & $0.00 \%$ & 0.869 & $2[51][56]$ & 334 \\
\hline
\end{tabular}


Table 2 (continued)

\begin{tabular}{|c|c|c|c|c|c|c|c|c|c|}
\hline Risk factor & Pooled OR & LCI & UCI & Chi-2 & Chi-2 $p$ value & $\mathrm{I} 2$ & $P$ value & No. of studies & $\begin{array}{l}\text { No. of } \\
\text { partic- } \\
\text { ipants }\end{array}$ \\
\hline $\begin{array}{l}\text { Previous radiation pneumo- } \\
\text { nitis (history of radiation } \\
\text { pneumonitis vs no history } \\
\text { of radiation pneumonitis) }\end{array}$ & 2.584 & 0.971 & 6.877 & 3.52 & 0.061 & $71.60 \%$ & 0.057 & 2 [29] [30] & 284 \\
\hline $\begin{array}{l}\text { Radiotherapy (previous radio- } \\
\text { therapy vs no radiotherapy) }\end{array}$ & 1.04 & 0.715 & 1.511 & 1.5 & 0.96 & $0.00 \%$ & 0.839 & $\begin{array}{l}7[26][29][31][32][48] \\
{[51][52]}\end{array}$ & 939 \\
\hline Sex (male vs female) & 1.033 & 0.904 & 1.182 & 23.15 & 0.185 & $22.20 \%$ & 0.631 & $\begin{array}{l}19[25][26][27][28][29] \\
{[30][31][32][33][34][46]} \\
{[47][48][49][51][53][54]} \\
{[56][58]}\end{array}$ & 5630 \\
\hline $\begin{array}{l}\text { Smoking pack-years }(\geq 50 \\
\text { vs }<50 \text { packs/year) }\end{array}$ & 0.994 & 0.981 & 1.007 & 0.12 & 0.726 & $0.00 \%$ & 0.368 & 3 [28] [32] [48] & 302 \\
\hline $\begin{array}{l}\text { Smoking status (current/for- } \\
\text { mer vs never smoker) }\end{array}$ & 1.249 & 1.021 & 1.528 & 6 & 0.916 & $0.00 \%$ & 0.031 & $\begin{array}{l}13[25][26][27][29][30] \\
{[31][33][46][49][53][54]} \\
{[56][58]}\end{array}$ & 3873 \\
\hline Stage (stage III/IV vs I/II) & 1.269 & 0.846 & 1.901 & 17.02 & 0.009 & $64.80 \%$ & 0.249 & $\begin{array}{l}7[26][32][51][53][54] \\
{[56][58]}\end{array}$ & 1499 \\
\hline $\begin{array}{l}\text { Combination treatment } \\
\text { (ICI + chemotherapy vs } \\
\text { monotherapy) }\end{array}$ & 0.686 & 0.439 & 1.073 & 5.4 & 0.067 & $62.90 \%$ & 0.099 & 3 [27] [54] [55] & 1128 \\
\hline $\begin{array}{l}\text { Treatment line (first vs sec- } \\
\text { ond/third line) }\end{array}$ & 1.039 & 0.792 & 1.363 & 6.64 & 0.467 & $0.00 \%$ & 0.782 & $\begin{array}{l}8 \text { [27] [32] [33] [48] [53] [58] } \\
\quad[56][49]\end{array}$ & 2654 \\
\hline $\begin{array}{l}\text { Treatment response (complete } \\
\text { response/partial response/ } \\
\text { stable disease vs progres- } \\
\text { sive disease) }\end{array}$ & 3.548 & 2.49 & 5.055 & 1.03 & 0.31 & $2.90 \%$ & 0.0001 & $2[54][56]$ & 761 \\
\hline $\begin{array}{l}\text { Use of steroids (previous use } \\
\text { of steroids vs no previous } \\
\text { use of steroids) }\end{array}$ & 1.58 & 0.976 & 2.556 & 12.17 & 0.002 & $83.60 \%$ & 0.063 & 3 [26] [27] [51] & 663 \\
\hline $\begin{array}{l}\text { White blood cell count }\left(\geq 10^{3}\right. \\
\left.\text { vs }<10^{3} / \mathrm{mm}^{3}\right)\end{array}$ & 1 & 1 & 1 & 3.84 & 0.05 & $74.00 \%$ & 0.35 & 2 [29] [48] & 253 \\
\hline
\end{tabular}

$O R$ odds ratio; $L C I$ lower confidence interval; $U C I$ upper confidence interval; $C O P D$ chronic obstructive pulmonary disease; $C R P \mathrm{C}$-reactive protein; $I L D$ interstitial lung disease; $N L R$ neutrophil-lymphocyte ratio; $P D$ - $L 1$ programmed death-ligand $1 ; L D H$ lactate dehydrogenase; $U / L$ units per liter; $m g / d L$ milligrams per deciliter

studies were pooled (Supplementary Table 4). For the other stratified analysis by study region, we found that region can be a source of heterogeneity for stage as a risk factor and the use of pembrolizumab. Significant heterogeneity was no longer observed for these risk factors when only studies from Asia were pooled (Supplementary Table 5).

Multiple risk factors were only reported by a single study, rendering them unsuitable for meta-analysis. The following risk factors were found to be statistically significantly related to the development of irAEs in one of the studies: a decrease in albumin level, the absence of concomitant irAEs a higher eosinophil count, a higher fibrosis score, imaging findings of airway obstruction adjacent to lung tumors (IAOT), a lower lung immune prognostic index, the presence of less than three organs affected by the cancer, the history of elevated autoantibodies, history of preexisting rheumatoid factor, history of previous interstitial pneumonia, a higher tumor burden, use of beta-blockers, use of loop diuretics, and use of taxanes (Supplementary Table 1). Finally, some studies reported risk factors for specific types of irAEs as a secondary outcome. In these studies, preexisting rheumatoid factor increased the risk of developing skin reactions, and women reported to be at a higher risk of developing endocrinopathies (Supplementary Table 6).

\section{Discussion}

In this meta-analysis including 25 studies and 6696 patients, several risk factors for the development of irAEs in patients with NSCLC treated with ICIs were identified. These factors were increased C-reactive protein level, increased NLR, ground glass attenuation on CT, use of PD-1 
CRP mg/dL

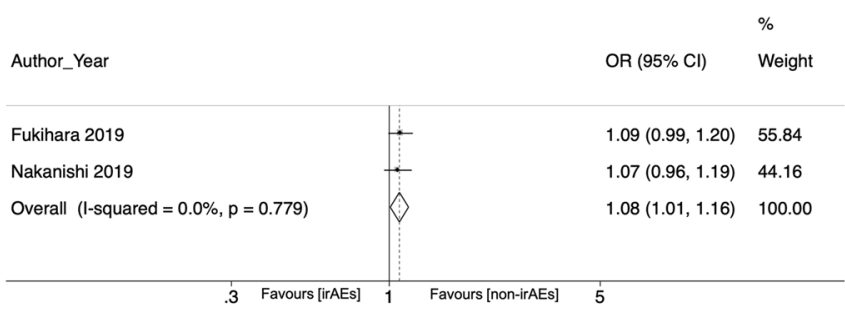

NLR

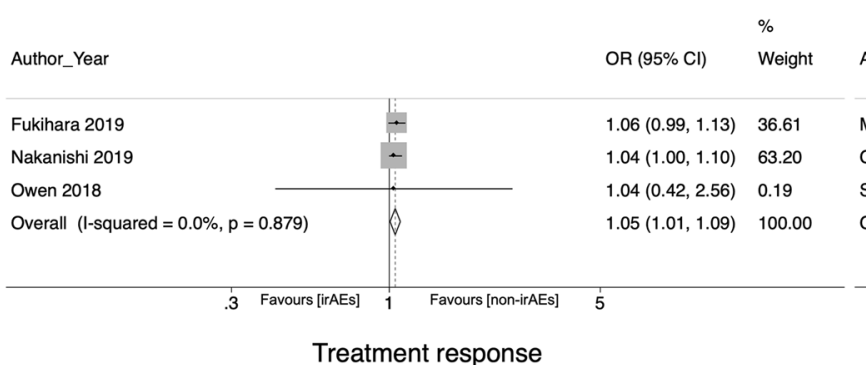

Treatment response
PD-L1 expression
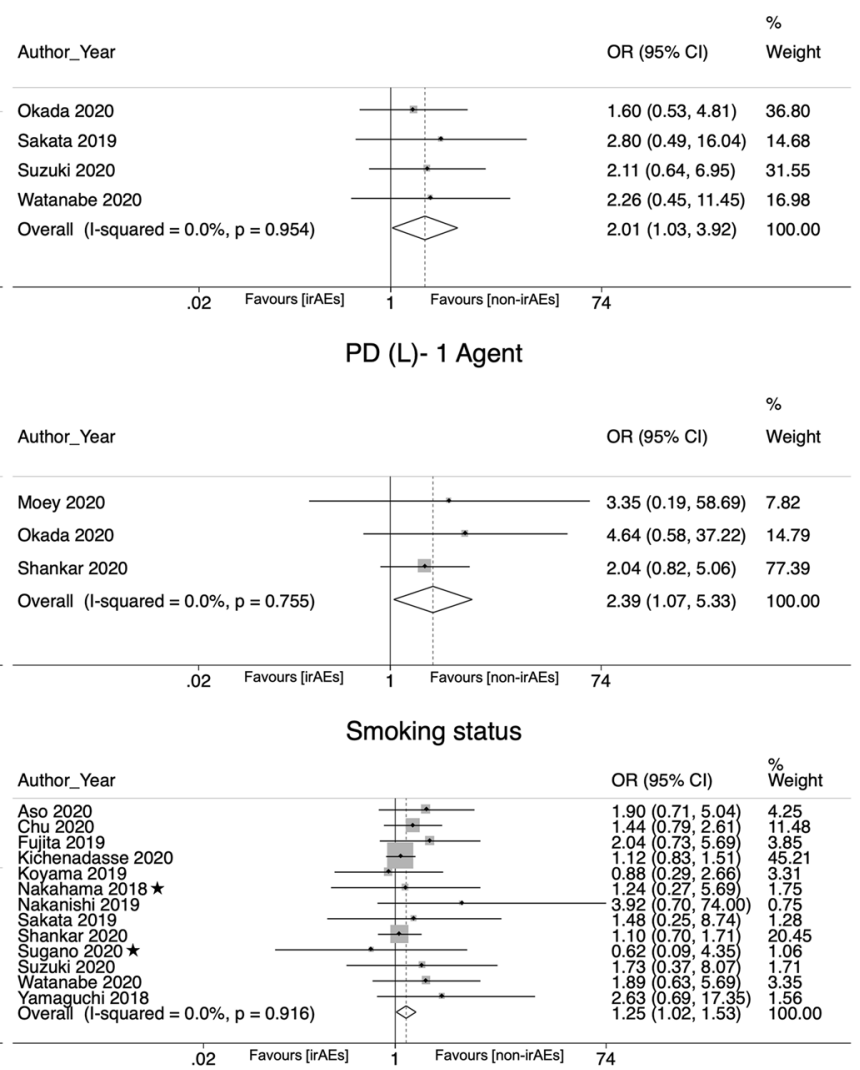

Ground glass attenuation

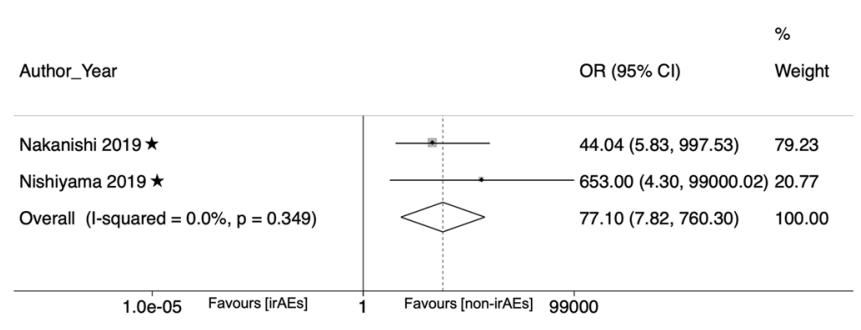

Fig. 1 Forest plots for random effect meta-analysis of the associations between potential risk factors and immune-related adverse events. $O R$ odds ratio; $C I$ confidence interval; $C R P$ C-reactive protein; NLR neutrophil-lymphocyte ratio. $\pitchfork$ Studies that provided adjusted odds ratios

inhibitor therapy, PD-L1 expression, smoking, and treatment response. These risk factors are mostly related to the inflammatory response or the treatment response. This illustrates that a predisposition to acute inflammatory response and/or poorer immune self-tolerance may be the underlying mechanisms explaining why the presence of the identified factors increases the risk of irAEs following treatment with ICIs in NSCLC patients.

First of all, the laboratory markers of inflammatory response were shown to be associated with a higher risk of irAEs. C-reactive protein and NLR are both markers of ongoing inflammatory processes, and stimulation of the immune system by ICIs can then further promote the immune response resulting in irAEs [35-37].

With regard to treatment and response, the identified risk factors are in line with what can be expected based on previous literature. The relationship between a better response to treatment and a greater occurrence of irAEs has been previously reported [38]. Related to this, a higher PD-L1 expression, a risk factor studied in this systematic review, has previously been shown to be associated with better treatment response [39]. As for the type of immune checkpoint targeted by ICIs, PD-1 inhibitors have been shown to have a worse safety profile than PD-L1 inhibitors [40].

Moreover, smoking was shown to be associated with a higher risk of irAEs, likely due to its impact on inflammation 
in the airway and lung parenchyma. Smoking is known to cause changes in the patterns of the normal immune response and inflammatory processes, as well as recruitment of autoantibodies [41, 42]. This enhancement in inflammatory response and recruitment of autoantibody-stimulated immune response cells in lung tissue can lead to a loss of self-tolerance and may contribute to the development of irAEs.

Furthermore, the presence of ground glass attenuation visible on CT images showed an association with patient's risk of irAEs. Ground glass attenuation can be a marker of pre-existing inflammatory activity and susceptibility to acute inflammatory response, that can further be enhanced by ICIs [29] [43].

Several of the risk factors presented in this review and meta-analysis are assessed as part of routine care in patients before the initiation of immunotherapy and/or as part of their follow-up. Some of these risk factors markedly increased the risk of developing irAEs, such as the presence of ground glass attenuation in CT. A favorable treatment response tripled the risk of irAEs. The use of PD-1 inhibitors as opposed to PD-L1 inhibitors, and a high expression of PD-L1 doubled the risk of irAEs. Other risk factors had a modest effect on the risk of irAEs, such as a history of smoking, high NLR and CRP values.

\section{Strengths and limitations}

The major strength of this study is the high level of homogeneity among studies; all studies shared the same study design and had similar patient inclusion criteria. Furthermore, in line with previous studies we found that overall, irAEs of interest were reported for $25 \%$ of the patients. However, since many studies focused on selected irAEs only (i.e., pneumonitis or interstitial lung disease), it is likely that this estimate is an underestimation of the percentage of patients experiencing irAEs of any type.

A main limitation of this study is that some risk factors may remain unexplored, since in several cases only one paper explored a specific risk factor, making it not possible to pool the results in the meta-analysis. Some of these single study-based risk factors excluded from the metaanalysis share a possible causal origin in the alterations of the immune system, such as a history of high levels of antibodies or rheumatoid factor. In the case of ground glass attenuation as a risk factor, the results should be interpreted with caution, since the study carried out by Nishiyama includes only patients with a history of having preexisting ILD, which could lead to a selection bias. Another limitation is that in a substantial proportion of the cases we extracted crude OR from the reported frequencies and as such we could not take potential confounding into account. For this, we also conducted analyses excluding these crude ORs, which lowered the number of risk factors that could be analyzed. All findings were comparable, with the exception of squamous histology of the tumor, being significant in the adjusted analysis only. Likewise, in the analyses stratified by type of irAE, we found that a worse performance status is a risk factor for developing severe irAEs, while the opposite was true for the main meta-analysis pooling studies irrespective of the type of irAE they reported as outcome.

Finally, another important aspect to take into account is the observed heterogeneity after pooling the results for some risk factors. In our sensitivity analysis, we stratified by region and study quality/risk of bias as assessed with QUIPS and found that region can explain part of the heterogeneity in the effect of stage as well as the use of pembrolizumab, and quality/risk of bias can contribute to heterogeneity in the case of stage and combination treatment. In these cases, heterogeneity $\left(I^{2}\right)$ decreased below $50 \%$ when stratified by region or quality/risk of bias.

\section{Implications and recommendations}

The risk factors identified in this review may help in selecting treatment regimen for patients at a higher risk of developing irAEs and could contribute to the decision of starting therapy with ICIs. When deciding to treat identified patients with a higher risk for irAEs, these patients should be monitored more closely. Early, adequate treatment of irAEs will result in a better clinical outcome [44]. Given the limited number of studies looking at the relationship between patient characteristics and the risk of irAEs, future studies are needed to explore determinants of irAEs and search for new mechanisms through which these risk factors contribute to the development of irAEs.

Supplementary Information The online version contains supplementary material available at https://doi.org/10.1007/s00262-021-02996-3.

Authors' contributions Suazo-Zepeda E. Formal analysis, Investigation, Data Curation, Writing-Original Draft, Visualization; Bokern $M$. Investigation, Data Curation, Writing—Review \& Editing; Vinke P.C. Conceptualization, Methodology, Validation, Data Curation, Writing-Review \& Editing, Supervision; Hiltermann T.J.N. Conceptualization, Methodology, Writing-Review \& Editing, Supervision; de Bock G.H. Conceptualization, Methodology, Writing-Review \& Editing, Supervision; Sidorenkov G. Conceptualization, Methodology, Validation, Data Curation, Writing-Review \& Editing, Supervision.

Funding This research did not receive any funding from funding agencies in the public, commercial, or not-for-profit sectors.

Data Availability The authors confirm that the data supporting the findings of this study are available within the supplementary materials. 


\section{Declaration}

Conflicts of interest The authors declare no conflict of interest.

Open Access This article is licensed under a Creative Commons Attribution 4.0 International License, which permits use, sharing, adaptation, distribution and reproduction in any medium or format, as long as you give appropriate credit to the original author(s) and the source, provide a link to the Creative Commons licence, and indicate if changes were made. The images or other third party material in this article are included in the article's Creative Commons licence, unless indicated otherwise in a credit line to the material. If material is not included in the article's Creative Commons licence and your intended use is not permitted by statutory regulation or exceeds the permitted use, you will need to obtain permission directly from the copyright holder. To view a copy of this licence, visit http://creativecommons.org/licenses/by/4.0/.

\section{References}

1. Bray F, Ferlay J, Soerjomataram I, Siegel RL, Torre LA, Jemal A (2018) Global cancer statistics 2018: GLOBOCAN estimates of incidence and mortality worldwide for 36 cancers in 185 countries. CA Cancer J Clin 68:394-424. https://doi.org/10.3322/caac. 21492

2. National Cancer Institute (2018) SEER cancer statistics review. 1975-2015. http://seer.cancer.gov/csr/1975_2015/

3. American Cancer Society (2019) Cancer facts \& figures 2019. Am Cancer Soc 76:105-108

4. Corrales L, Scilla K, Caglevic C, Miller K, Oliveira J, Rolfo C (2018) Immunotherapy in lung cancer: a new age in cancer treatment. In: Naing A, Hajjar J (eds) Immunotherapy. Springer International Publishing, Cham, pp 65-95. https://doi.org/10.1007/ 978-3-030-02505-2_3

5. Jain P, Jain C, Velcheti V (2018) Role of immune-checkpoint inhibitors in lung cancer. Ther Adv Respir Dis 12:1753465817750075. https://doi.org/10.1177/1753465817 750075

6. Sharon E, Streicher H, Goncalves P, Chen HX (2014) Immune checkpoint inhibitors in clinical trials. Chin J Cancer 33:434-444. https://doi.org/10.5732/cjc.014.10122

7. Antonia SJ, Borghaei H, Ramalingam SS, Horn L, De Castro CJ, Pluzanski A et al (2019) Four-year survival with nivolumab in patients with previously treated advanced non-small-cell lung cancer: a pooled analysis. Lancet Oncol 20:1395-1408. https:// doi.org/10.1016/S1470-2045(19)30407-3

8. Antonia SJ, Villegas A, Daniel D, Vicente D, Murakami S, Hui $R$ et al (2017) Durvalumab after chemoradiotherapy in stage III non-small-cell lung cancer. N Engl J Med 377:1919-1929. https:// doi.org/10.1056/nejmoa1709937

9. Gadgeel S, Rodríguez-Abreu D, Speranza G, Esteban E, Felip E, Dómine M, Hui R, Hochmair MJ, Clingan P, Powell SF, Cheng SY, Bischoff HG, Peled N, Grossi F, Jennens RR, Reck M, Garon EB, Novello S, Rubio-Viqueira B,Boyer M, Kurata T, Gray JE, Yang J, Bas T, Pietanza MC, Garassino MC (2020) Updated analysis from KEYNOTE-189:pembrolizumab or placebo plus pemetrexed and platinum for previously untreated metastatic nonsquamous nonsmall-cell lung cancer. J Clin Oncol 38(14):1505-1517. https:// doi.org/10.1200/JCO.19.03136

10. Paz-Ares L, Vicente D, Tafreshi A, Robinson A, Soto Parra H, Mazières $\mathrm{J}$ et al (2020) A randomized, placebo-controlled trial of pembrolizumab plus chemotherapy in patients with metastatic squamous NSCLC: protocol-specified final analysis of
KEYNOTE-407. J Thorac Oncol 15:1657-1669. https://doi.org/ 10.1016/j.jtho.2020.06.015

11. Reck M, Rodríguez-Abreu D, Robinson AG, Hui R, Csoszi T, Fülöp A et al (2019) Updated analysis of KEYNOTE-024: pembrolizumab versus platinum-based chemotherapy for advanced non-small-cell lung cancer with PD-L1 tumor proportion score of 50\% or greater. J Clin Oncol 37:537-546. https://doi.org/10. 1200/JCO.18.00149

12. Socinski MA, Jotte RM, Cappuzzo F, Orlandi F, Stroyakovskiy D, Nogami N et al (2018) Atezolizumab for first-line treatment of metastatic nonsquamous NSCLC. N Engl J Med 378:2288-2301. https://doi.org/10.1056/nejmoa1716948

13. Spain L, Diem S, Larkin J (2016) Management of toxicities of immune checkpoint inhibitors. Cancer Treat Rev 44:51-60. https://doi.org/10.1016/j.ctrv.2016.02.001

14. Wang Q, Xu R (2019) Immunotherapy-related adverse events (irAEs): extraction from FDA drug labels and comparative analysis. JAMIA Open 2:173-178. https://doi.org/10.1093/jamiaopen/ ooy045

15. Michot JM, Bigenwald C, Champiat S, Collins M, Carbonnel F, Postel-Vinay $S$ et al (2016) Immune-related adverse events with immune checkpoint blockade: a comprehensive review. Eur J Cancer 54:139-148. https://doi.org/10.1016/j.ejca.2015.11.016

16. Tartari F, Santoni M, Burattini L, Mazzanti P, Onofri A, Berardi R (2016) Economic sustainability of anti-PD-1 agents nivolumab and pembrolizumab in cancer patients: recent insights and future challenges. Cancer Treat Rev 48:20-24. https://doi.org/10.1016/j.ctrv.2016.06.002

17 Haanen JBAG, Carbonnel F, Robert C, Kerr KM, Peters S, Larkin J et al (2017) Management of toxicities from immunotherapy: ESMO clinical practice guidelines for diagnosis, treatment and follow-up. Ann Oncol Off J Eur Soc Med Oncol 28:iv119-iv142. https://doi.org/10.1093/annonc/mdx225

18. Brahmer JR, Lacchetti C, Schneider BJ, Atkins MB, Brassil $\mathrm{KJ}$, Caterino JM et al (2018) Management of immune-related adverse events in patients treated with immune checkpoint inhibitor therapy: American society of clinical oncology clinical practice guideline. J Clin Oncol Off J Am Soc Clin Oncol 36:1714-1768. https://doi.org/10.1200/JCO.2017.77.6385

19. Thapa B, Roopkumar J, Kim AS, Gervaso L, Patil PD, Calabrese $C$ et al (2019) Incidence and clinical pattern of immune related adverse effects (irAE) due to immune checkpoint inhibitors (ICI). J Clin Oncol 37:e14151-e14151. https://doi.org/10. 1200/JCO.2019.37.15_suppl.e14151

20. Khoja L, Day D, Wei-Wu Chen T, Siu LL, Hansen AR (2017) Tumour- and class-specific patterns of immune-related adverse events of immune checkpoint inhibitors: a systematic review. Ann Oncol 28:2377-2385. https://doi.org/10.1093/annonc/ $\operatorname{mdx} 286$

21. Wang DY, Salem J-E, Cohen JV, Chandra S, Menzer C, Ye F et al (2018) Fatal toxic effects associated with immune checkpoint inhibitors: a systematic review and meta-analysis. JAMA Oncol 4:1721-1728. https://doi.org/10.1001/jamaoncol.2018.3923

22. Centre for Reviews and Dissemination (2016) Guidance notes for registering a systematic review protocol with PROSPERO. University of York, pp 3-23

23. The Cochrane Collaboration (2021) Collecting data-form for RCTs and non-RCTs, pp 1-22

24. Hayden JA, van der Windt DA, Cartwright JL, Côté P, Bombardier C (2013) Assessing bias in studies of prognostic factors. Ann Intern Med 158:280-286. https://doi.org/10.7326/0003-4819-1584-201302190-00009

25. Koyama J, Horiike A, Yoshizawa T, Dotsu Y, Ariyasu R, Saiki $\mathrm{M}$ et al (2019) Correlation between thyroid transcription factor-1 expression, immune-related thyroid dysfunction, and efficacy of anti-programmed cell death protein-1 treatment in non-small 
cell lung cancer. J Thorac Dis 11:1919-1928. https://doi.org/10. 21037/jtd.2019.04.102

26. Fujita K, Kim YH, Kanai O, Yoshida H, Mio T, Hirai T (2019) Emerging concerns of infectious diseases in lung cancer patients receiving immune checkpoint inhibitor therapy. Respir Med 146:66-70. https://doi.org/10.1016/j.rmed.2018.11.021

27. Chu X, Zhao J, Zhou J, Zhou F, Jiang T, Jiang S et al (2020) Association of baseline peripheral-blood eosinophil count with immune checkpoint inhibitor-related pneumonitis and clinical outcomes in patients with non-small cell lung cancer receiving immune checkpoint inhibitors. Lung Cancer 150:76-82. https:// doi.org/10.1016/j.lungcan.2020.08.015

28. Nishiyama N, Honda T, Sema M, Kawahara T, Jin Y, Natsume I et al (2020) The utility of ground-glass attenuation score for anticancer treatment-related acute exacerbation of interstitial lung disease among lung cancer patients with interstitial lung disease. Int J Clin Oncol 25:282-291. https://doi.org/10.1007/ s10147-019-01576-x

29. Nakanishi Y, Masuda T, Yamaguchi K, Sakamoto S, Horimasu Y, Nakashima T et al (2019) Pre-existing interstitial lung abnormalities are risk factors for immune checkpoint inhibitor-induced interstitial lung disease in non-small cell lung cancer. Respir Investig 57:451-459. https://doi.org/10.1016/j.resinv.2019.05.002

30. Nakahama K, Tamiya A, Isa S-I, Taniguchi Y, Shiroyama T, Suzuki $\mathrm{H}$ et al (2018) Association between imaging findings of airway obstruction adjacent to lung tumors and the onset of interstitial lung disease after nivolumab. Vivo 32:887-891. https://doi.org/10.21873/invivo.11324

31. Sugano T, Seike M, Saito Y, Kashiwada T, Terasaki Y, Takano $\mathrm{N}$ et al (2020) Immune checkpoint inhibitor-associated interstitial lung diseases correlate with better prognosis in patients with advanced non-small-cell lung cancer. Thorac Cancer 11:10521060. https://doi.org/10.1111/1759-7714.13364

32. Okada N, Matsuoka R, Sakurada T, Goda M, Chuma M, Yagi K et al (2020) Risk factors of immune checkpoint inhibitor-related interstitial lung disease in patients with lung cancer: a singleinstitution retrospective study. Sci Rep 10:1-9. https://doi.org/ 10.1038/s41598-020-70743-2

33. Yamaguchi T, Shimizu J, Hasegawa T, Horio Y, Inaba Y, Yatabe $\mathrm{Y}$ et al (2018) Pre-existing pulmonary fibrosis is a risk factor for anti-PD-1-related pneumonitis in patients with nonsmall cell lung cancer: a retrospective analysis. Lung Cancer 125:212-217. https://doi.org/10.1016/j.lungcan.2018.10.001

34. Duma N, Abdel-Ghani A, Yadav S, Hoversten KP, Reed CT, Sitek AN et al (2019) Sex differences in tolerability to anti-programmed cell death protein 1 therapy in patients with metastatic melanoma and non-small cell lung cancer: are we all equal? Oncologist 24:e1148-e1155. https://doi.org/10.1634/theoncolog ist.2019-0094

35. Nehring SM, Goyal A, Bansal P, Patel BC (2020) C reactive protein. StatPearls Publishing, Treasure island (FL), StatPearls

36. Forget $\mathrm{P}$, Khalifa C, Defour J-P, Latinne D, Van Pel M-C, De Kock M (2017) What is the normal value of the neutrophil-tolymphocyte ratio? BMC Res Notes 10:12. https://doi.org/10. 1186/s13104-016-2335-5

37. Zahorec R (2001) Ratio of neutrophil to lymphocyte countsrapid and simple parameter of systemic inflammation and stress in critically ill. Bratisl Lek Listy 102:5-14

38. Das S, Johnson DB (2019) Immune-related adverse events and anti-tumor efficacy of immune checkpoint inhibitors. J Immunother Cancer 7:1-11. https://doi.org/10.1186/ s40425-019-0805-8

39. Xu Y, Wan B, Chen X, Zhan P, Zhao Y, Zhang T et al (2019) The association of PD-L1 expression with the efficacy of antiPD-1/PD-L1 immunotherapy and survival of non-small cell lung cancer patients: a meta-analysis of randomized controlled trials. Transl Lung Cancer Res 8:413-428. https://doi.org/10. 21037/tlcr.2019.08.09

40. Huang YF, Xie WJ, Fan HY, Du J (2019) Comparative safety of PD-1/PD-L1 inhibitors for cancer patients: systematic review and network meta-analysis. Front Oncol 9:1-15. https://doi.org/ 10.3389/fonc.2019.00972

41. Strzelak A, Ratajczak A, Adamiec A, Feleszko W (2018) Tobacco smoke induces and alters immune responses in the lung triggering inflammation, allergy, asthma and other lung diseases: a mechanistic review. Int J Environ Res Public Health 15:1033. https://doi.org/10.3390/ijerph15051033

42. Hogg JC (2006) Why does airway inflammation persist after the smoking stops? Thorax 61:96-97. https://doi.org/10.1136/thx. 2005.049502

43. Gao J-W, Rizzo S, Ma L-H, Qiu X-Y, Warth A, Seki N et al (2017) Pulmonary ground-glass opacity: computed tomography features, histopathology and molecular pathology. Transl Lung Cancer Res Small Pulm Nodules 6(1):68-75

44. Naing A, Hajjar J, Gulley JL, Atkins MB, Ciliberto G, MericBernstam F et al (2020) Strategies for improving the management of immune-related adverse events. J Immunother Cancer 8:1-9. https://doi.org/10.1136/jitc-2020-001754

45. Ahmed T, Lycan T, Dothard A, Ehrlichman P, Ruiz J, Farris M et al (2020) Performance status and age as predictors of immunotherapy outcomes in advanced non-small-cell lung cancer. Clin Lung Cancer 21:e286-e293. https://doi.org/10.1016/j.cllc. 2020.01.001

46. Aso M, Toi Y, Sugisaka J, Aiba T, Kawana S, Saito R et al (2020) Association between skin reaction and clinical benefit in patients treated with anti-programmed cell death 1 monotherapy for advanced non-small cell lung cancer. Oncologist 25:e536e544. https://doi.org/10.1634/theoncologist.2019-0550

47. Cortellini A, Friedlaender A, Banna GL, Porzio G, Bersanelli M, Cappuzzo F et al (2020) Immune-related adverse events of pembrolizumab in a large real-world cohort of patients with NSCLC with a PD-L1 expression $\geq 50 \%$ and their relationship with clinical outcomes. Clin Lung Cancer 21:498-508.e2. https://doi.org/10.1016/j.cllc.2020.06.010

48. Fukihara J, Sakamoto K, Koyama J, Ito T, Iwano S, Morise $M$ et al (2019) Prognostic impact and risk factors of immunerelated pneumonitis in patients with non-small-cell lung cancer who received programmed death 1 inhibitors. Clin Lung Cancer 20:442-450.e4. https://doi.org/10.1016/j.cllc.2019.07.006

49. Kichenadasse G, Miners JO, Mangoni AA, Rowland A, Hopkins AM, Sorich MJ (2020) Multiorgan immune-related adverse events during treatment with atezolizumab. JNCCN J Natl Compr Cancer Netw 18:1191-1199. https://doi.org/10.6004/ jncen.2020.7567

50. Metro G, Banna GL, Signorelli D, Gili A, Galetta D, Galli G et al (2020) Efficacy of pembrolizumab monotherapy in patients with or without brain metastases from advanced non-small cell lung cancer with a PD-L1 expression $\geq 50 \%$. J Immunother 43:299-306. https://doi.org/10.1097/CJI.0000000000000340

51. Moey MYY, Tomdio AN, McCallen JD, Vaughan LM, O'Brien $\mathrm{K}$, Naqash AR et al (2020) Characterization of immune checkpoint inhibitor-related cardiotoxicity in lung cancer patients from a rural setting. JACC Cardio Oncol 2:491-502. https:// doi.org/10.1016/j.jaccao.2020.07.005

52. Owen DH, Wei L, Bertino EM, Edd T, Villalona-Calero MA, He $\mathrm{K}$ et al (2018) Incidence, risk factors, and effect on survival of immune-related adverse events in patients with non-small-cell lung cancer. Clin Lung Cancer 19:e893-900. https://doi.org/10. 1016/j.cllc.2018.08.008

53. Sakata Y, Kawamura K, Ichikado K, Shingu N, Yasuda Y, Eguchi Y et al (2019) The association between tumor burden and severe immune-related adverse events in non-small cell lung 
cancer patients responding to immune-checkpoint inhibitor treatment. Lung Cancer 130:159-161. https://doi.org/10.1016/j. lungcan.2019.02.011

54. Shankar B, Zhang J, Naqash AR, Forde PM, Feliciano JL, Marrone KA et al (2020) Multisystem immune-related adverse events associated with immune checkpoint inhibitors for treatment of non-small cell lung cancer. JAMA Oncol. https://doi. org/10.1001/jamaoncol.2020.5012

55. Suresh K, Voong KR, Shankar B, Forde PM, Ettinger DS, Marrone KA et al (2018) Pneumonitis in non-small cell lung cancer patients receiving immune checkpoint immunotherapy: incidence and risk factors. J Thorac Oncol 13:1930-1939. https:// doi.org/10.1016/j.jtho.2018.08.2035

56. Suzuki Y, Karayama M, Uto T, Fujii M, Matsui T, Asada K et al (2020) Assessment of immune-related interstitial lung disease in patients with NSCLC treated with immune checkpoint inhibitors: a multicenter prospective study. J Thorac Oncol 15:13171327. https://doi.org/10.1016/j.jtho.2020.04.002
57. Toi Y, Sugawara S, Sugisaka J, Ono H, Kawashima Y, Aiba T et al (2019) Profiling preexisting antibodies in patients treated with anti-PD-1 therapy for advanced non-small cell lung cancer. JAMA Oncol 5:376-383. https://doi.org/10.1001/jamaoncol. 2018.5860

58. Watanabe S, Ota T, Hayashi M, Ishikawa H, Otsubo A, Shoji S et al (2020) Prognostic significance of the radiologic features of pneumonitis induced by anti-PD-1 therapy. Cancer Med 9:3070-3077. https://doi.org/10.1002/cam4.2974

59. Zeng X, Zhu S, Xu C, Wang Z, Su X, Zeng D et al (2020) Effect of comorbidity on outcomes of patients with advanced nonsmall cell lung cancer undergoing anti-PD1 immunotherapy. Med Sci Monit 26:1-7. https://doi.org/10.12659/MSM.922576

Publisher's Note Springer Nature remains neutral with regard to jurisdictional claims in published maps and institutional affiliations. 